\title{
Endoscopic Ultrasound-Guided Biliary Drainage for Benign Biliary Diseases
}

\author{
Yousuke Nakai ${ }^{1}$, Hirofumi Kogure ${ }^{1}$, Hiroyuki Isayama ${ }^{2}$ and Kazuhiko Koike ${ }^{1}$ \\ Department of Gastroenterology, Graduate School of Medicine, ${ }^{1}$ The University of Tokyo, Tokyo, ${ }^{2}$ Juntendo University, Tokyo, Japan
}

\begin{abstract}
Although endoscopic retrograde cholangiopancreatography (ERCP) is the first-line treatment for benign biliary diseases, this procedure is technically difficult in some conditions such as a surgically altered anatomy and gastric outlet obstruction. After a failed ERCP, a surgical or a percutaneous approach is selected as a rescue procedure; however, various endoscopic ultrasound (EUS)-guided interventions are increasingly utilized in pancreatobiliary diseases, including EUS-guided rendezvous for failed biliary cannulation, EUS-guided antegrade treatment for stone management, and EUS-guided hepaticogastrostomy for anastomotic strictures in patients with a surgically altered anatomy. There are some technical hurdles in EUS-guided interventions for benign biliary diseases owing to the difficulty in puncturing a relatively small bile duct and in subsequent guidewire manipulation, as well as the lack of dedicated devices. A recent major advancement in this field is the introduction of a 2-step approach, in which EUS-guided drainage is placed in the first session and antegrade treatment is performed in subsequent sessions. This approach allows the use of various techniques such as mechanical lithotripsy and cholangioscopy without a risk of bile leak. In summary, EUS-guided interventions are among the treatment options for benign biliary diseases; however, standardization of the procedure and development of a treatment algorithm are needed.

Clin Endosc 2019;52:212-219
\end{abstract}

Key Words: Benign biliary diseases; Biliary drainage; Cholangioscopy; Endoscopic ultrasound

\section{INTRODUCTION}

Endoscopic retrograde cholangiopancreatography (ERCP) has been utilized for the diagnosis and treatment of biliary tract diseases, both benign and malignant. However, ERCP is technically difficult in some conditions such as a surgically altered anatomy ${ }^{1}$ and gastric outlet obstruction. ${ }^{2}$ Percutaneous transhepatic biliary drainage (PTBD) has been used as a salvage technique for failed ERCP; however, it needs more than 2-4 weeks of tube placement and is associated with high morbidity.

Received: October 19, 2018 Revised: December 5, 2018

Accepted: December 5, 2018

Correspondence: Yousuke Nakai

Department of Gastroenterology, Graduate School of Medicine, The University of Tokyo, 7-3-1 Hongo Bunkyo-ku, Tokyo 113-8655, Japan

Tel: +81-3-3815-5411, Fax: +81-3-5800-9801, E-mail: ynakai-tky@umin.ac.jp ORCID: https://orcid.org/0000-0001-7411-1385

(cc) This is an Open Access article distributed under the terms of the Creative Commons Attribution Non-Commercial License (http://creativecommons.org/ licenses/by-nc/3.0) which permits unrestricted non-commercial use, distribution, and reproduction in any medium, provided the original work is properly cited.
Endoscopic ultrasound (EUS) was first developed as a new diagnostic modality and is now widely used for tissue acquisition in various intra-abdominal lesions (i.e., EUS-guided fine-needle aspiration [EUS-FNA]). ${ }^{3,4}$ With EUS-FNA, EUS-guided interventions have evolved from being diagnostic procedures to being therapeutic procedures. EUS-guided biliary drainage (EUS-BD) is one of those EUS-guided therapeutic interventions. EUS-BD was first reported in 2001 by Giovannini et al., ${ }^{5}$ and since then has been increasingly performed mostly in patients with unresectable malignant biliary obstruction. ${ }^{6,7}$ However, as EUS can be utilized for biliary access, the indications of EUS-BD are not limited to malignant biliary obstruction and the use of EUS-BD for benign biliary diseases is increasingly reported. ${ }^{8-12}$

In this review, the current evidences and hurdles in the use of EUS-BD for benign biliary diseases are reviewed. We did not include EUS-guided gallbladder drainage (EUS-GBD) in our review because systematic reviews of EUS-GBD have already been reported elsewhere. ${ }^{13,14}$ 


\section{INDICATIONS OF EUS-BD IN BENIGN BILIARY DISEASES}

The indication and types of EUS-BD are summarized in Table 1 . Bile duct stones and benign biliary strictures are 2 major indications of EUS-BD in benign biliary diseases. There are 2 types of EUS-BD procedures: transpapillary (or trans-anastomotic) procedures and transmural biliary drainage. The former includes EUS-guided rendezvous (EUS-RV) and EUS-guided antegrade treatment (EUS-AG), and the latter includes EUS-guided choledochoduodenostomy, EUS-guided hepaticogastrostomy (EUS-HGS), EUS-guided hepaticojejunostomy (EUS-HJS), and EUS-GBD. EUS-BD for benign biliary diseases is currently used as a rescue procedure after a failed or difficult ERCP. The contraindications include lack of biliary tract visualization, presence of intervening vessels, severe coagulopathy, and presence of massive ascites. ${ }^{15}$

In benign biliary diseases other than cholecystitis, EUS-RV, EUS-AG, and EUS-HGS/HJS are 3 major methods for EUSBD. EUS-RV is performed for failed biliary cannulation in patients with a regular anatomy. EUS-AG is performed for stone removal in patients with a surgically altered anatomy. EUSHGS/HJS is performed for anastomotic strictures in patients with a surgically altered anatomy. The techniques and outcomes of these 3 EUS-BD interventions are discussed below.

\section{EUS-RV}

In EUS-RV, after the puncture of the biliary tract, a guidewire is passed through the ampulla into the duodenum or through the anastomosis into the jejunum. Then, by using this RV guidewire, biliary cannulation is achieved in an ERCP fashion. Subsequently, intended procedures such as stone extraction and biliary drainage are performed.

EUS-RV is indicated when failed cannulation in a regular anatomy is the reason for EUS-BD. As subsequent procedures are similar to the regular ERCP, EUS-RV can be performed both for bile duct stones and benign biliary strictures.

In a systematic review, ${ }^{16}$ the technical success rate of EUSRV was $82 \%$ (85\% in the extrahepatic approach and $76 \%$ in the intrahepatic approach), which appears lower than the $>90 \%$ technical success rate of EUS-guided transmural biliary drainage. ${ }^{17}$ This technical difficulty of EUS-RV lies in its complex steps, especially guidewire manipulation. ${ }^{18}$ This guidewire passage can be further difficult in patients with benign biliary strictures, which necessitate passage through both the stricture and the ampulla/anastomosis.

There are 3 routes in EUS-RV in patients with a regular anatomy: transgastric route, transduodenal route in a long position, and transduodenal route in a short position. ${ }^{19}$ The transduodenal route in a short position is recommended whenever possible because of its easy guidewire manipulation. $^{18,20}$ There are some techniques that can enhance the technical success of EUS-RV, such as the hybrid technique ${ }^{21}$ and the hitch-and-ride cannulation technique. ${ }^{22}$

The adverse event rate of EUS-RV was $13 \%$ in a systematic review. ${ }^{16}$ Adverse events include bleeding, perforation, bile leak, pancreatitis, abdominal pain, and pneumoperitoneum. The adverse event rate was $8 \%$ in the extrahepatic approach and $17 \%$ in the intrahepatic approach. Thus, the extrahepatic approach, especially the transduodenal route in a short position, should be selected whenever possible for safety and efficacy.

In patients with a surgically altered anatomy, EUS-RV can be performed if the ampulla or the anastomosis is accessible by an enteroscope; ${ }^{23}$ however, scope insertion in patients with a surgically altered anatomy may be cumbersome. Therefore, as discussed below, EUS-AG or the transmural approach can be utilized in the treatment of benign biliary diseases. Conversely, EUS-RV in combination with enteroscopy-assisted ERCP might be necessary when EUS-AG stone treatment fails. $^{24,25}$ Thus, devices for EUS-RV, EUS-AG, and EUS-HGS should be prepared in all situations.

Table 1. Indications and Types of EUS-BD for Benign Biliary Diseases

\begin{tabular}{lll}
\hline Reasons for interventions & \multicolumn{1}{c}{ Reasons for EUS-BD } & Types of EUS-BD procedures \\
\hline Bile duct stones & Failed cannulation & EUS-RV \\
Benign biliary strictures & Failed access to the ampulla or the anastomosis & EUS-AG stone treatment \\
Bile leak & Failed guidewire passage through the stricture & EUS-HGS \\
Cholecystitis & Clinical failure after ERCP & EUS-HJS \\
& & EUS-GBD \\
\hline
\end{tabular}

ERCP, endoscopic retrograde cholangiopancreatography; EUS-AG, endoscopic ultrasound-guided antegrade treatment; EUS-BD, endoscopic ultrasound-guided biliary drainage; EUS-GBD, endoscopic ultrasound-guided gallbladder drainage; EUS-HGS, endoscopic ultrasound-guided hepaticogastrostomy; EUS-HJS, endoscopic ultrasound-guided epaticojejunostomy; EUS-RV, endoscopic ultrasound-guided rendezvous. 


\section{EUS-AG STONE EXTRACTION IN A SURGICALLY ALTERED ANATOMY}

Endoscopic management of bile duct stones in patients with a surgically altered anatomy is still a problem despite the recent advancements in enteroscopy-assisted ERCP. ${ }^{1,26}$ Although the technical success rate of enteroscopy-assisted ERCPs is more than $95 \%$ when performed by experts, ${ }^{27}$ technical failure may occur in enteroscope insertion or in biliary cannulation depending on the postsurgical adhesion or the length of the afferent limb. While PTBD has been used as a salvage procedure after failed ERCP in this setting, ${ }^{28}$ EUS-AG stone extraction is increasingly reported as another salvage technique (Table 2).

In EUS-AG stone extraction, biliary access is achieved through the puncture of an intrahepatic bile duct, followed by guidewire advancement through the ampulla into the duodenum. Then, balloon dilation of the ampulla is performed, and bile duct stones are antegradely pushed out with an extraction balloon catheter. After complete stone extraction, a temporary nasobiliary drainage tube or a plastic stent is often placed to prevent bile leak.

Data on EUS-AG stone removal are still limited. Two early single-center studies demonstrated technical success rates of $67 \%(4 / 6)^{24}$ and $60 \%(3 / 5) .{ }^{25}$ The success rate did not improve considerably when only simple stone extraction techniques were used: 1 multicenter retrospective study of EUS-AG stone extraction in patients with a surgically altered anatomy with a native papilla demonstrated a technical success rate of $72 \%$ and an adverse event rate of $17 \%{ }^{29}$ The reasons for technical failure were failed bile duct puncture (21\%), failed guidewire manipulation (3\%), and failed stone extraction (3\%).

The technical difficulty of EUS-AG stone extraction lies in every step of the procedure. The dilation of the intrahepatic bile duct is often minimal as compared with a large common bile duct in patients with a surgically altered anatomy, which explains the relatively high rate of failed puncture. Guidewire passage is also difficult owing to the long distance from the intrahepatic bile duct to the ampulla. Finally, stone extraction is also technically difficult because of the limited available devices for stone extraction.

In EUS-AG, ampullary intervention is limited to papillary balloon dilation up to the size of the distal bile duct. ${ }^{30}$ Therefore, if stones are larger than the size of the dilation balloon, lithotripsy is necessary, which may prolong the procedure and increase the risk of bile leak.

To overcome these limitations of EUS-AG stone removal, a 2-step approach can be a treatment option. ${ }^{31}$ In the first session, a plastic stent or a covered metallic stent is placed via EUS-HGS or EUS-HIS without stone removal. Then, in the subsequent sessions after fistula maturation, antegrade stone removal is performed. Because of the mature fistula, there is a low risk of bile leak. Moreover, various devices can be used, such as a mechanical lithotriptor or even a peroral cholangioscope, which can be inserted through the bilio-enteric fistula, allowing the use of electrohydraulic lithotripsy or laser lithotripsy under direct visualization. Hosmer et al. ${ }^{32}$ reported their single-center experience of 2-step EUS-AG stone extraction in 9 patients with a surgically altered anatomy. Stone extraction was completed at a mean of 2.5 sessions with cholangioscopy $(n=8)$, electrohydraulic lithotripsy $(n=4)$, and mechanical lithotripsy $(n=1)$. The use of a temporary fully covered metallic stent $^{33}$ allows retrograde stone extraction in addition to passing various devices through the stent. The use of fully covered metallic stents was reported in 2 other studies, and stent removal after stone extraction was successful in $100 \%{ }^{32,34}$ The best approach to EUS-guided bile duct stone management should be further investigated according to the anatomy and the size and location of bile duct stones.

\section{EUS-HGS/HJS FOR BENIGN BILIARY STRICTURES IN A SURGICALLY ALTERED ANATOMY}

The development of an anastomotic stricture after hepaticojejunostomy, which can be a cause of cholangitis or jaundice, is not uncommon, and a long-standing anastomotic stricture with cholangitis can lead to secondary cirrhosis if left untreated. Owing to the surgically altered anatomy, endoscopic management of the anastomotic stricture is technically challenging. Recently, the safety and efficacy of enteroscopy-assisted therapeutic ERCP have been reported; ${ }^{27}$ however, it is not always technically and clinically successful even when performed by experts. In cases of failed enteroscopy-assisted ERCP, PTBD or (less often) a surgical approach has been utilized as a rescue procedure, although EUS- $\mathrm{BD}^{35-37}$ is now another treatment option (Table 2). In most cases with a surgically altered anatomy, the EUS-guided approach is limited to the left biliary system. The right biliary system is approachable through the EUS-HGS or EUS-HJS route in cases with choledochojejunostomy. Otherwise, the right biliary system should be managed with PTBD or enteroscopy-assisted ERCP. If the anastomosis is accessible by ERCP, a combination of enteroscopy and the EUS approach is also useful: ERCP for the right biliary drainage and EUS for the left biliary drainage.

Miranda-Garcia et al. ${ }^{38}$ reported their pilot study of EUSHGS in 7 cases with anastomotic strictures. In 4 cases, a guidewire could pass the stricture and stents were placed across the stricture for anastomotic dilation. Meanwhile, in 


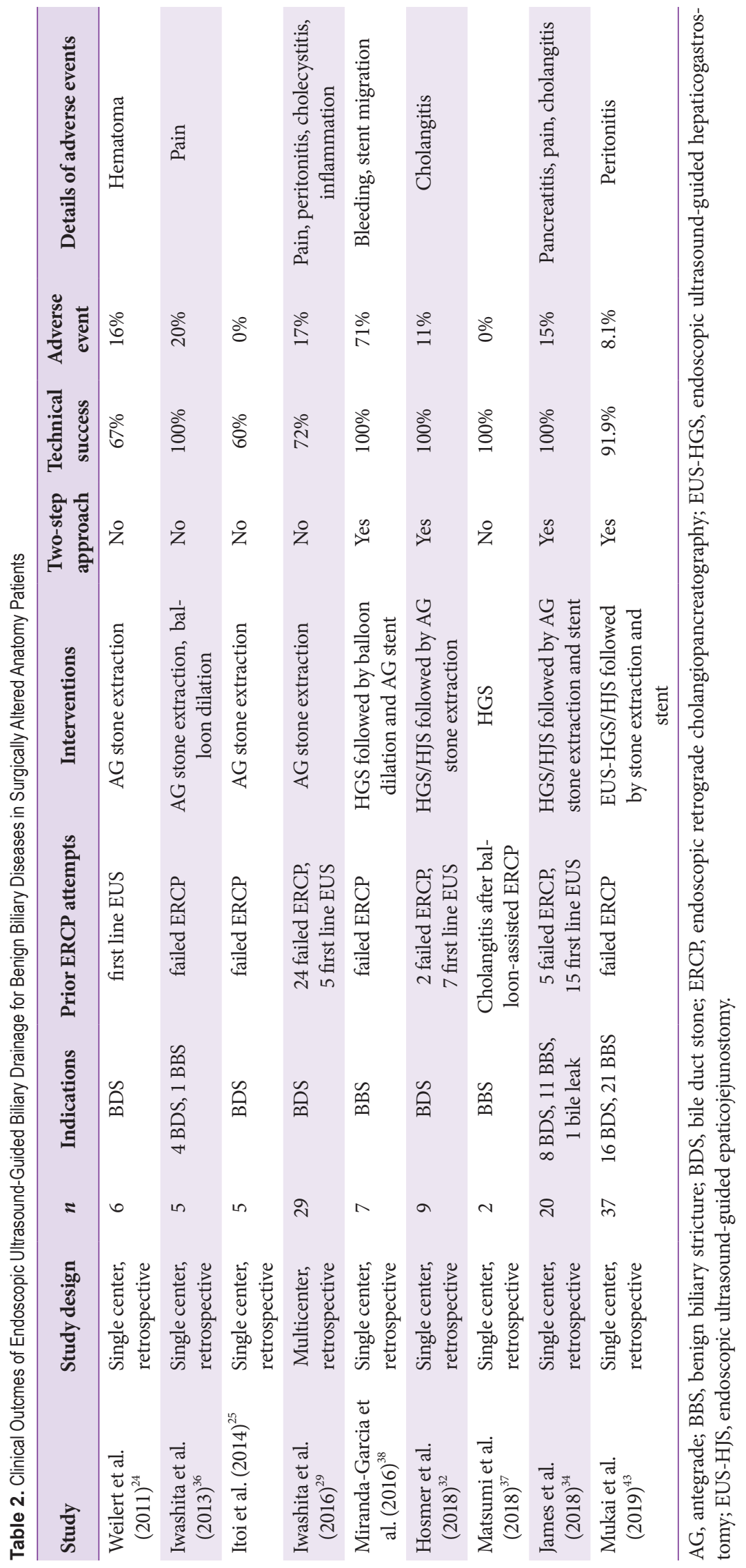


the remaining 3 cases, a guidewire could not pass the stricture and only EUS-HGS was performed. A fully covered metallic stent was placed via EUS-HGS in 6 cases. The technical and clinical success rates were $57 \%$ and $100 \%$, respectively. There were 3 bleeding cases and 3 stent migration cases as adverse events.

In cases with a tight anastomotic stricture, we prefer a 2-step approach similar to EUS-AG stone extraction. ${ }^{31}$ In the first session, EUS-HGS or EUS-HJS is performed. After fistula maturation, the anastomosis can be passed with a hydrophilic guidewire or even with cholangioscopy guidance through the fistula. Once a stent is placed through the anastomosis via the EUS approach, stent exchange either through the entero-biliary fistula or with an enteroscopic approach, if accessible, is technically possible. In general, however, stent exchange through the EUS-HGS fistula is technically easier than enteroscopy-assisted ERCP. Recently, a fully covered metallic stent is reported to be useful for benign biliary strictures during ERCP ${ }^{39,40}$ In EUS-guided interventions, a fully covered metallic stent with a large diameter may provide better stricture resolution; however, no large-scale data comparing plastic and covered metallic stents are available yet.

\section{TECHNICAL TIPS FOR EUS-GUIDED TREATMENT OF BENIGN BILIARY DISEASES}

One of the technical hurdles in EUS-guided interventions for benign biliary diseases is the lack of dedicated devices.

Both puncture of an intrahepatic bile duct and guidewire insertion are difficult in most cases with a surgically altered anatomy because of minimal dilation of the intrahepatic bile duct. Given the lack of a dedicated biliary access needle, we recommend the use of a 19-gauge FNA needle with a sharp tip (EZshot 3 plus; Olympus Medical Systems, Tokyo, Japan) and a 0.025 -inch guidewire with a flexible tip (VisiGlide2, Olympus Medical Systems). There is a risk of guidewire shearing within the small intrahepatic bile duct, ${ }^{41}$ and gentle guidewire manipulation is mandatory. Even after successful guidewire insertion into the biliary system, subsequent guidewire passage through the ampulla, anastomosis, and/or stricture can also be difficult. To avoid guidewire shearing, once the guidewire is advanced deep into the biliary system, the FNA needle is exchanged to a tapered catheter. Then, further guidewire manipulation can be safely and effectively performed.

As compared with the transpapillary approach, which maintains physiological bile flow, EUS-BD can be complicated by bile leak. A prolonged procedure in the initial EUS-BD session can increase the risk of bile leak. When a complex proce- dure is planned, a 2-step procedure after fistula maturation allows the use of various devices without the risk of bile leak. In addition, the use of a covered metallic stent allows easy access to the bile duct. ${ }^{33}$ There are no comparative data on adverse events after EUS-BD for benign vs. malignant diseases. One systematic review of EUS-BD reported an adverse event rate of $17 \%$, ${ }^{42}$ which appeared to be similar to that of EUS-BD for benign biliary diseases shown in Table 2 . However, given the variety of EUS-BD procedures, further investigations on the safety of EUS-BD for benign biliary diseases are warranted.

The greatest advancement in EUS-AG treatment is the use of a peroral cholangioscope through the EUS-guided bilio-enteric fistula. Management of bile duct stones and biliary strictures under direct visualization of a cholangioscope provides a high technical success rate. In a recent report of EUSAG interventions by Mukai et al., ${ }^{43}$ 2-step procedures were performed in 26 of 34 cases of benign biliary diseases. Among those 26 cases, peroral cholangioscopy through the bilio-enteric fistula was utilized in 19 cases. Hosmer et al. ${ }^{32}$ also utilized cholangioscopy in 8 of 9 cases of bile duct stones in patients with a surgically altered anatomy. Given the possibilities of EUS-BD as a portal to various intraductal interventions, a combination of EUS-BD and peroral cholangioscopy through the bilio-enteric fistula can be a standard technique for biliary interventions in patients with a surgically altered anatomy.

\section{PROPOSAL FOR A TREATMENT ALGORITHM IN BENIGN BILIARY DISEASES}

Our proposal for a treatment algorithm in benign biliary diseases is shown in Fig. 1. EUS-guided interventions are currently considered a salvage technique after failed ERCP; however, as shown in Table 2, it can be a first-line treatment in patients with a surgically altered anatomy if expertise in EUS-BD is available. ${ }^{32,34}$ In patients with a surgically altered anatomy, the puncture site is limited to the left intrahepatic bile duct and PTBD should be selected for those who need interventions in the right biliary system. Otherwise, EUS-guided interventions have an advantage over PTBD in terms of clinical success and adverse events. ${ }^{17}$ When EUS-guided treatment is unlikely to be completed in a single session because of difficult stones or a tight stricture, a 2-step approach should be selected.

Given the potential severe adverse events such as bile leak, bleeding, and perforation, the treatment selection of EUS-guided intervention vs. enteroscopy-assisted ERCP or vs. PTBD should be decided according to the anatomy and the local expertise. Last but not least, support from the mul- 


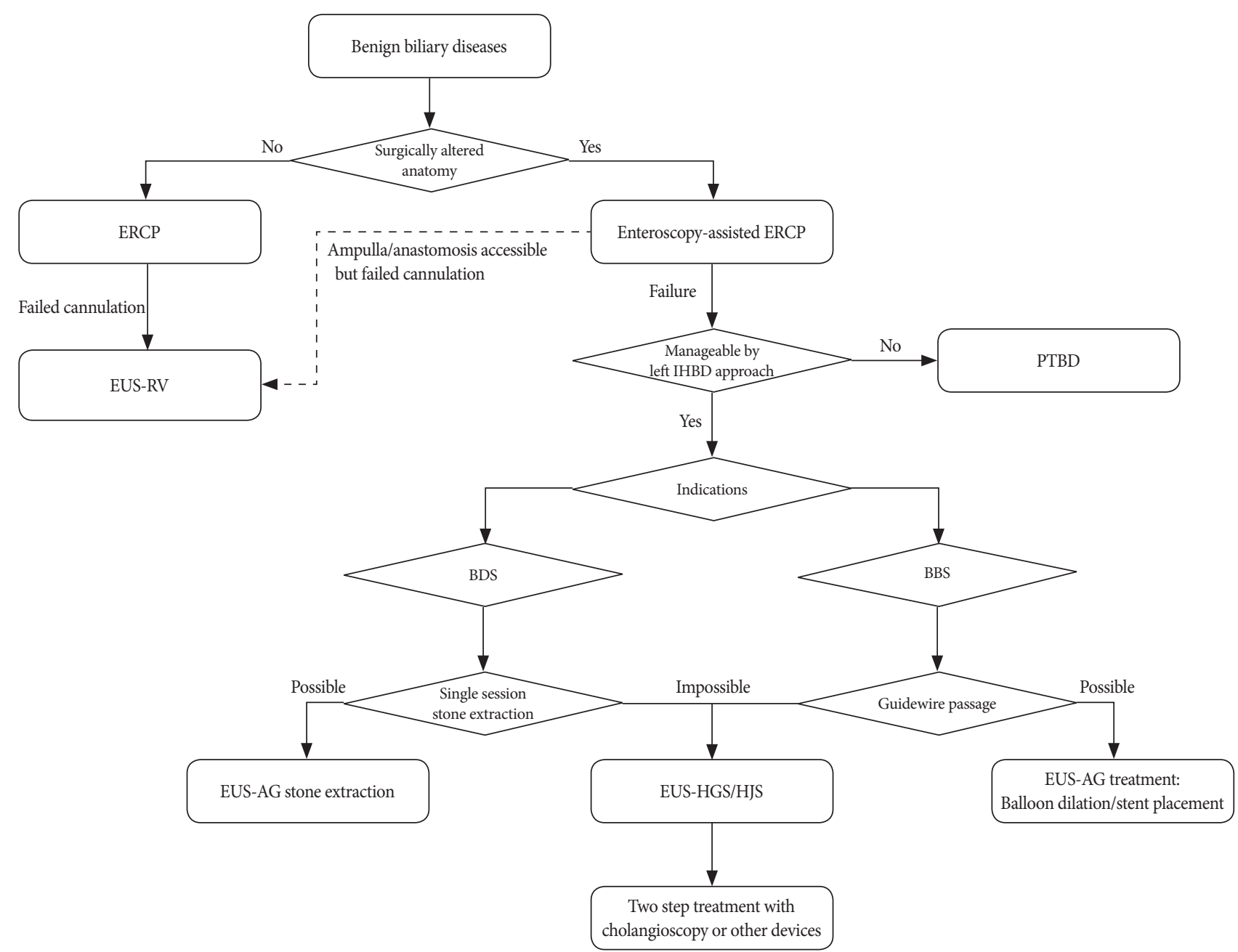

Fig. 1. Proposal for a treatment algorithm for benign biliary diseases. BBS, benign biliary stricture; BDS, bile duct stones; ERCP, endoscopic retrograde cholangiopancreatography; EUS-AG, endoscopic ultrasound-guided antegrade treatment; EUS-HGS, endoscopic ultrasound-guided hepaticogastrostomy; EUS-HJS, endoscopic ultrasound-guided epaticojejunostomy; EUS-RV, endoscopic ultrasound-guided rendezvous; IHBD, intrahepatic bile duct; PTBD, percutaneous transhepatic biliary drainage.

tidisciplinary team including endoscopists, interventional radiologists, and surgeons should be readily available when EUS-guided intervention fail or adverse events occur. ${ }^{15}$

\section{LIMITATIONS OF EUS-BD}

There are some limitations in the EUS-BD approach for benign biliary diseases. First, dedicated devices are definitely lacking in the EUS-BD approach. This lack of dedicated devices has been discussed since the introduction of EUS-BD, but the problem is yet to be resolved. There are some reasons for this slow development of dedicated devices, including the lack of procedure standardization and the variety of the procedures, as described above, within the spectrum of EUS-BD. Moreover, from the point view of manufacturers, the number of procedures is limited owing to the expertise necessary for EUS-BD. Given the increasing number of EUS-BD cases as well as the development of training models, the development of dedicated EUS devices by manufacturers will hopefully accelerate, which would, in turn, bring benefits to patients. Given the minimal dilation of the intrahepatic bile duct in patients with a surgically altered anatomy, the development of an access needle, which enables easy puncture and guidewire manipulation without shearing, is necessary. The appropriate stent for EUS-BD in benign biliary diseases is also unclear. While there are fewer adverse events with covered metallic stents than with plastic stents in EUS-BD, ${ }^{6}$ stents should be removable in benign diseases. Recently, the safety and effectiveness of a dedicated plastic stent for EUS-BD ${ }^{44}$ were reported. However, when EUS-BD is used as a portal to intraductal interventions such as lithotripsy under cholangioscopy, a large- 
bore fully covered metallic stent provides better access. Meanwhile, given the cost of fully covered metallic stents, their role in EUS-BD for benign biliary diseases should further be investigated in comparison with plastic stents. Finally, the indication and selection of procedures, including EUS and/ or enteroscopy-assisted therapeutic procedures, vary among different institutions. ${ }^{8}$ The indication and algorithm for EUS$\mathrm{BD}$ in benign biliary diseases should be established based on large-scale prospective data. Finally, the procedures for benign biliary diseases are more complex than those of EUS-BD for malignant biliary obstruction, and it is difficult to manage all conditions with EUS-BD alone; here, both ERCP and PTBD are complimentary to EUS-BD.

\section{CONCLUSIONS}

In conclusion, EUS-guided biliary interventions such as EUS-RV, EUS-AG, and EUS-HGS are now among the treatment options for benign biliary diseases, which are difficult to manage with conventional methods. The introduction of a 2-step approach and the use of a cholangioscope through the bilio-enteric fistula are the major breakthroughs; however, standardization of each EUS-BD procedure and the development of a treatment algorithm in this setting are needed.

Conflicts of Interest

The authors have no financial conflicts of interest.

\section{REFERENCES}

1. Nakai Y, Kogure H, Yamada A, Isayama H, Koike K. Endoscopic management of bile duct stones in patients with surgically altered anatomy. Dig Endosc 2018;30 Suppl 1:67-74.

2. Nakai Y, Hamada T, Isayama H, Itoi T, Koike K. Endoscopic management of combined malignant biliary and gastric outlet obstruction. Dig Endosc 2017;29:16-25.

3. Vilmann P, Jacobsen GK, Henriksen FW, Hancke S. Endoscopic ultrasonography with guided fine needle aspiration biopsy in pancreatic disease. Gastrointest Endosc 1992;38:172-173.

4. Chang KJ, Albers CG, Erickson RA, Butler JA, Wuerker RB, Lin F. Endoscopic ultrasound-guided fine needle aspiration of pancreatic carcinoma. Am J Gastroenterol 1994;89:263-266.

5. Giovannini M, Moutardier V, Pesenti C, Bories E, Lelong B, Delpero JR. Endoscopic ultrasound-guided bilioduodenal anastomosis: a new technique for biliary drainage. Endoscopy 2001;33:898-900.

6. Kawakubo $\mathrm{K}$, Isayama $\mathrm{H}$, Kato $\mathrm{H}$, et al. Multicenter retrospective study of endoscopic ultrasound-guided biliary drainage for malignant biliary obstruction in Japan. J Hepatobiliary Pancreat Sci 2014;21:328-334.

7. Nakai Y, Isayama $\mathrm{H}$, Yamamoto N, et al. Safety and effectiveness of a long, partially covered metal stent for endoscopic ultrasound-guided hepaticogastrostomy in patients with malignant biliary obstruction. Endoscopy 2016;48:1125-1128.

8. Nakai Y, Isayama H, Yamamoto N, et al. Indications for endoscopic ultrasonography (EUS)-guided biliary intervention: does EUS always come after failed endoscopic retrograde cholangiopancreatography? Dig Endosc 2017;29:218-225.

9. Ryozawa S, Fujita N, Irisawa A, Hirooka Y, Mine T. Current status of interventional endoscopic ultrasound. Dig Endosc 2017;29:559-566.

10. Paik WH, Park DH. Endoscopic ultrasound-guided biliary access, with focus on technique and practical tips. Clin Endosc 2017;50:104-111.

11. Saumoy M, Kahaleh M. Safety and complications of interventional endoscopic ultrasound. Clin Endosc 2018;51:235-238.

12. Minaga K, Kitano M. Recent advances in endoscopic ultrasound-guided biliary drainage. Dig Endosc 2018;30:38-47.

13. Choi JH, Lee SS. Endoscopic ultrasonography-guided gallbladder drainage for acute cholecystitis: from evidence to practice. Dig Endosc 2015;27:1-7.

14. Peñas-Herrero I, de la Serna-Higuera C, Perez-Miranda M. Endoscopic ultrasound-guided gallbladder drainage for the management of acute cholecystitis (with video). J Hepatobiliary Pancreat Sci 2015;22:35-43.

15. Teoh AYB, Dhir V, Kida M, et al. Consensus guidelines on the optimal management in interventional EUS procedures: results from the Asian EUS group RAND/UCLA expert panel. Gut 2018;67:1209-1228.

16. Tsuchiya T, Itoi T, Sofuni A, Tonozuka R, Mukai S. Endoscopic ultrasonography-guided rendezvous technique. Dig Endosc 2016;28 Suppl 1:96-101.

17. Sharaiha RZ, Khan MA, Kamal F, et al. Efficacy and safety of EUS-guided biliary drainage in comparison with percutaneous biliary drainage when ERCP fails: a systematic review and meta-analysis. Gastrointest Endosc 2017;85:904-914.

18. Iwashita T, Yasuda I, Mukai T, et al. EUS-guided rendezvous for difficult biliary cannulation using a standardized algorithm: a multicenter prospective pilot study (with videos). Gastrointest Endosc 2016;83:394-400.

19. Kawakubo K, Isayama H, Sasahira N, et al. Clinical utility of an endoscopic ultrasound-guided rendezvous technique via various approach routes. Surg Endosc 2013;27:3437-3443.

20. Iwashita T, Lee JG, Shinoura S, et al. Endoscopic ultrasound-guided rendezvous for biliary access after failed cannulation. Endoscopy 2012;44:60-65.

21. Iwashita T, Uemura S, Yoshida K, et al. EUS-guided hybrid rendezvous technique as salvage for standard rendezvous with intra-hepatic bile duct approach. PLoS One 2018;13:e0202445.

22. Nakai $Y$, Isayama $H$, Matsubara S, et al. A novel "hitch-and-ride" deep biliary cannulation method during rendezvous endoscopic ultrasound-guided ERCP technique. Endoscopy 2017;49:983-988.

23. Matsunami Y, Itoi T, Tsuchiya T, Tonozuka R, Mukai S. EUS-guided rendezvous technique for refractory benign biliary stricture caused by postoperative bile-duct injury. VideoGIE 2017;2:326-327.

24. Weilert F, Binmoeller KF, Marson F, Bhat Y, Shah JN. Endoscopic ultrasound-guided anterograde treatment of biliary stones following gastric bypass. Endoscopy 2011;43:1105-1108.

25. Itoi T, Sofuni A, Tsuchiya T, Ijima M, Iwashita T. Endoscopic ultrasonography-guided transhepatic antegrade stone removal in patients with surgically altered anatomy: case series and technical review (with videos). J Hepatobiliary Pancreat Sci 2014;21:E86-E93.

26. Moreels TG. Update in enteroscopy: new devices and new indications. Dig Endosc 2018;30:174-181.

27. Shimatani M, Hatanaka H, Kogure H, et al. Diagnostic and therapeutic endoscopic retrograde cholangiography using a short-type double-balloon endoscope in patients with altered gastrointestinal anatomy: a multicenter prospective study in Japan. Am J Gastroenterol 2016;111:17501758.

28. Moon JH, Cho YD, Ryu CB, et al. The role of percutaneous transhepatic papillary balloon dilation in percutaneous choledochoscopic lithotomy. Gastrointest Endosc 2001;54:232-236.

29. Iwashita T, Nakai Y, Hara K, Isayama H, Itoi T, Park DH. Endoscopic ultrasound-guided antegrade treatment of bile duct stone in patients with surgically altered anatomy: a multicenter retrospective cohort study. J 
Hepatobiliary Pancreat Sci 2016;23:227-233.

30. Itoi T, Ryozawa S, Katanuma A, et al. Japan Gastroenterological Endoscopy Society guidelines for endoscopic papillary large balloon dilation. Dig Endosc 2018;30:293-309.

31. Nakai Y, Isayama H, Koike K. Two-step endoscopic ultrasonography-guided antegrade treatment of a difficult bile duct stone in a surgically altered anatomy patient. Dig Endosc 2018;30:125-127.

32. Hosmer A, Abdelfatah MM, Law R, Baron TH. Endoscopic ultrasound-guided hepaticogastrostomy and antegrade clearance of biliary lithiasis in patients with surgically-altered anatomy. Endosc Int Open 2018;6:E127-E130.

33. Iwashita T, Yasuda I, Mukai T, et al. Successful management of biliary stones in the hepatic duct after a Whipple procedure by using an EUS-guided antegrade approach and temporary metal stent placement. Gastrointest Endosc 2014;80:337.

34. James TW, Fan YC, Baron TH. EUS-guided hepaticoenterostomy as a portal to allow definitive antegrade treatment of benign biliary diseases in patients with surgically altered anatomy. Gastrointest Endosc 2018;88:547-554.

35. Jirapinyo P, Lee LS. Endoscopic ultrasound-guided pancreatobiliary endoscopy in surgically altered anatomy. Clin Endosc 2016;49:515-529.

36. Iwashita T, Yasuda I, Doi S, et al. Endoscopic ultrasound-guided antegrade treatments for biliary disorders in patients with surgically altered anatomy. Dig Dis Sci 2013;58:2417-2422.

37. Matsumi A, Kato H, Saragai Y, et al. Endoscopic ultrasound-guid- ed hepaticogastrostomy is effective for repeated recurrent cholangitis after surgery: two case reports. Case Rep Gastrointest Med 2018;2018:7201967.

38. Miranda-Garcia P, Gonzalez JM, Tellechea JI, Culetto A, Barthet M. EUS hepaticogastrostomy for bilioenteric anastomotic strictures: a permanent access for repeated ambulatory dilations? Results from a pilot study. Endosc Int Open 2016;4:E461-E465.

39. Coté GA, Slivka A, Tarnasky P, et al. Effect of covered metallic stents compared with plastic stents on benign biliary stricture resolution: a randomized clinical trial. JAMA 2016;315:1250-1257.

40. Zheng $\mathrm{X}, \mathrm{Wu}$ J, Sun B, et al. Clinical outcome of endoscopic covered metal stenting for resolution of benign biliary stricture: systematic review and meta-analysis. Dig Endosc 2017;29:198-210.

41. Ogura T, Masuda D, Takeuchi T, Fukunishi S, Higuchi K. Liver impaction technique to prevent shearing of the guidewire during endoscopic ultrasound-guided hepaticogastrostomy. Endoscopy 2015;47:E583-E584.

42. Khan MA, Akbar A, Baron TH, et al. Endoscopic ultrasound-guided biliary drainage: a systematic review and meta-analysis. Dig Dis Sci 2016;61:684-703.

43. Mukai S, Itoi T, Sofuni A, et al. EUS-guided antegrade intervention for benign biliary diseases in patients with surgically altered anatomy (with videos). Gastrointest Endosc 2019;89:399-407.

44. Umeda J, Itoi T, Tsuchiya $\mathrm{T}$, et al. A newly designed plastic stent for EUS-guided hepaticogastrostomy: a prospective preliminary feasibility study (with videos). Gastrointest Endosc 2015;82:390-396.e2. 